\title{
Improved methanol-derived lovastatin production through enhancement of the biosynthetic pathway and intracellular lovastatin efflux in methylotrophic yeast
}

\author{
Yiqi Liu', Chenxiao Bai ${ }^{1}$, Qin Xu1, Jiahui Yu ${ }^{1}$, Xiangshan Zhou ${ }^{1}$, Yuanxing Zhang ${ }^{1,2}$ and Menghao Cai ${ }^{*}$
}

\begin{abstract}
Background: Methanol has attracted interest as a substrate for improvement of product titers and yields of bioprocesses, because methanol has a more reduced state than sugars do and can be renewably synthesized from abundant natural gas supplies. Our aim was to engineer methylotrophic Pichia pastoris to convert methanol into value-added lovastatin more productively.

Results: A strengthened biosynthetic pathway of lovastatin was constructed through the assembly of three modules with increasing module-specific antibiotic stress in the methylotrophic yeast $P$. pastoris. The resulting strain $(P$. p/LV_V\#9) produced $287.5 \pm 2.0 \mathrm{mg} / \mathrm{L}$ lovastatin in a 5 -L bioreactor from methanol. The production was further improved by identification and overexpression of a statin pump protein, TapA, a membrane protein capable of lovastatin efflux out of the cell. A TapA-overexpressing strain, P. p/LV_V\#9-TapA, produced $419.0 \pm 9.5 \mathrm{mg} / \mathrm{L}$ lovastatin from methanol: 46\% more than P. p/LV_V\#9 did, and 520\% more relative to the strain (P. p/LV_SC) with single-copy genes.

Conclusions: A methylotrophic yeast strain producing $419.0 \pm 9.5 \mathrm{mg} / \mathrm{L}$ lovastatin was constructed by optimization of biosynthetic gene dosages and coexpression of a statin pump protein; these results proved that $P$. pastoris is a promising chassis organism for natural-product biosynthesis. A membrane protein, TapA was found to perform the function of exporting intracellular lovastatin and enhanced lovastatin production.
\end{abstract}

Keywords: Enhanced biosynthetic pathway, Lovastatin, Methanol, Pichia pastoris, Statin pump protein

\section{Background}

Methanol, one of the $\mathrm{C} 1$ compounds, has attracted considerable interest as a promising feedstock for the production of value-added biological products (Bennett et al. 2018). Methanol can be inexpensively manufactured from natural gas (which is abundant in the deep sea of our planet) and can also be renewably obtained through reduction of carbon dioxide (Räuchle et al. 2016). Meanwhile, methanol possesses a higher degree of reduction than traditional sugars do (Whitaker et al. 2015). Although efforts have been focused on engineering

\footnotetext{
*Correspondence: cmh022199@ecust.edu.cn

1 State Key Laboratory of Bioreactor Engineering, East China University of Science and Technology, 130 Meilong Road, Shanghai 200237, China Full list of author information is available at the end of the article
}

native or synthetic methylotrophs to convert methanol for the production of biochemical metabolites, the effects are still at the rudimentary level owing to the limited genetic tools for native methylotrophs and the weak methanol consumption abilities of the artificial modified methylotrophs (Whitaker et al. 2015; Price et al. 2016). Pichia pastoris is a natural methylotrophic yeast that can utilize methanol for growth to high cell densities (Yang and Zhang 2017). It has been researched for years, and a variety of genetic tools and expression vectors has been commercialized (Cregg et al. 2000). Therefore, P. pastoris is well suited as a chassis cellular system to convert methanol to various value-added compounds (Schwarzhans et al. 2017). 
Lovastatin, a natural polyketide drug isolated from Aspergillus terreus, is used to treat hypercholesterolemia owing to its (3S)-hydroxy-3-methylglutaryl-coenzyme A (HMG-CoA) reductase-inhibitory activity (Campbell and Vederas 2010). Industrial lovastatin is mainly produced by fungal fermentation; however, fermentation using native $A$. terreus usually poses some problems such as a long culture period, difficult manipulation, and multiple byproducts (Vogl et al. 2013; Billingsley et al. 2016). With the development of synthetic biology, reconstruction of biosynthetic pathways in chassis organisms has been proved to be a possible solution to these problems (Ro et al. 2006; Galanie et al. 2015). The biosynthetic gene cluster and catalytic role of each functional gene product are well characterized for lovastatin (Kennedy et al. 1999; Xie et al. 2006; Barriuso et al. 2011; Xu et al. 2013). Previously, we assembled the biosynthetic pathway of lovastatin in the methylotrophic yeast $P$. pastoris (Fig. 1) and successfully produced lovastatin from methanol (Liu et al. 2018). Nonetheless, gene dosages in the lovastatinproducing strains were all maintained at single-copy levels, and a titer of only $250.8 \mathrm{mg} / \mathrm{L}$ lovastatin was obtained via an optimal coculture strategy (Liu et al. 2018); this titer is still much lower than that of a native lovastatinproducing strain (Singh and Pandey 2013; Huang et al. 2017).

For the biosynthesis of chemicals in a heterologous host, increased gene dosage is commonly used to strengthen pathways (Billingsley et al. 2016). Nonetheless, overexpressed heterologous genes may impose overwhelming metabolic stress on the host cell, while excessive intracellular accumulation of bioactive compounds may be toxic to the cell (Wang et al. 2017). Fungal secondary-metabolite gene clusters often contain a gene encoding a protein to pump the produced compounds out of cells, which thereby acts as a self-resistance mechanism (Martín et al. 2005). Previously, efflux pump protein MlcE was identified in Penicillium citrinum, and lovastatin resistance was observed in Saccharomyces cerevisiae overexpressing $m l c E$ (Ley et al. 2015). Because the lovastatin biosynthetic pathway had not yet been assembled in yeast (Ley et al. 2015), whether overexpression of $m l c E$ could improve lovastatin production was not determined. Additionally, whether this protein functions as an efflux pump to transport intracellular lovastatin remains unclear.

This work was aimed at improving lovastatin production by means of methanol as a feedstock through strengthening of the biosynthetic pathway and screening for high gene dosage setups in three continuous modules. Moreover, to alleviate the toxicity or metabolic stress resulting from the product (lovastatin), a statin pump protein was identified in a lovastatin-producing strain of
A. terreus and overexpressed to discharge the intracellular lovastatin. Finally, the producing strains were evaluated in a 5-L bioreactor to confirm the effectiveness of the strategies.

\section{Methods}

Strains, media, and cultivation conditions

Pichia pastoris GS115 (Invitrogen) served as a wild-type strain, grown at $30{ }^{\circ} \mathrm{C}$ in the YPD medium $[1 \%(\mathrm{w} / \mathrm{v})$ yeast extract, $2 \%(\mathrm{w} / \mathrm{v})$ peptone, $2 \%(\mathrm{w} / \mathrm{v})$ glucose] or the minimal YNB medium $[1.34 \%(\mathrm{w} / \mathrm{v})$ yeast nitrogen base without amino acids] supplemented with different carbon sources: $1 \%(\mathrm{w} / \mathrm{v})$ glycerol (YNG medium) or $0.5 \%$ (v/v) methanol (YNM medium). Escherichia coli Top10 cells were used for plasmid propagation and were incubated at $37{ }^{\circ} \mathrm{C}$ in the Luria-Bertani (LB) medium $[0.5 \%$ $(\mathrm{w} / \mathrm{v})$ yeast extract, $1 \%(\mathrm{w} / \mathrm{v})$ tryptone, and $0.5 \%(\mathrm{w} / \mathrm{v})$ $\mathrm{NaCl}$. A terreus (NIH 2624) was a source of the cDNA of tap $A$ and was incubated at $28{ }^{\circ} \mathrm{C}$ in the YM medium $[0.3 \%(\mathrm{w} / \mathrm{v})$ yeast extract, $0.3 \%(\mathrm{w} / \mathrm{v})$ malt extract, $0.5 \%$ $(\mathrm{w} / \mathrm{v})$ tryptone, and $2 \%(\mathrm{w} / \mathrm{v})$ glucose]. The solid medium was prepared by the addition of $2 \%$ agar into the liquid medium. The media were sterilized at $121^{\circ} \mathrm{C}$ for $20 \mathrm{~min}$; methanol was added separately, and a glucose solution was added separately after filtration sterilization. Antibiotics phleomycin D1 $\left(\right.$ Zeocin $\left.^{\mathrm{TM}}\right)$, geneticin 418 sulfate, and hygromycin B, purchased from Yeasen Biotech Co., Ltd. (China), were used to screen the strains.

\section{Construction of plasmids and strains}

Plasmids pZ_BCGN, pK_sAR, and pG_sDF were constructed as previously described (Liu et al. 2018). pZ BCGN was linearized by digestion with $B \ln \mathrm{I}$ and was transferred into $P$. pastoris GS115 by electroporation in accordance with the manufacturer's instructions (Invitrogen). The yeast recombinants were screened on YPD plates containing Zeocin at $0.1,0.5,1.0$, and $2.0 \mathrm{mg} / \mathrm{mL}$ to obtain dihydromonacolin $\mathrm{L}$ (DML)-producing strains (P. $p / \mathrm{DML})$. pK_sAR was linearized by digestion with SalI and transferred into $P . p / \mathrm{DML} \_\# 9$ by the same methods, and the transformants were screened on YPD plates containing G418 at $0.25,0.5,1.0$, and $2.0 \mathrm{mg} / \mathrm{mL}$ to obtain monacolin $\mathrm{J}(\mathrm{MJ})$-producing strains $(P . p / \mathrm{MJ})$. pG_sDF was linearized by $B \ln \mathrm{I}$ digestion and transferred into P. $p / \mathrm{MJ} \_\# 9$, and the transformants were screened on YPD plates containing hygromycin at $0.75,1.5,3.0$, and $5.0 \mathrm{mg} / \mathrm{mL}$ to obtain lovastatin ( $\mathrm{LV}$ )-producing strains $(P . p / \mathrm{LV})$. The genotypes of the recombinant strains were verified by PCR analysis.

Oligonucleotides were synthesized by Suzhou Genewiz Biotech Co., Ltd. (China). Fungal genomic DNA was extracted using a Plant Genomic DNA Extraction Kit (Tiangen Biotech Co., Ltd., China). Commercialized 


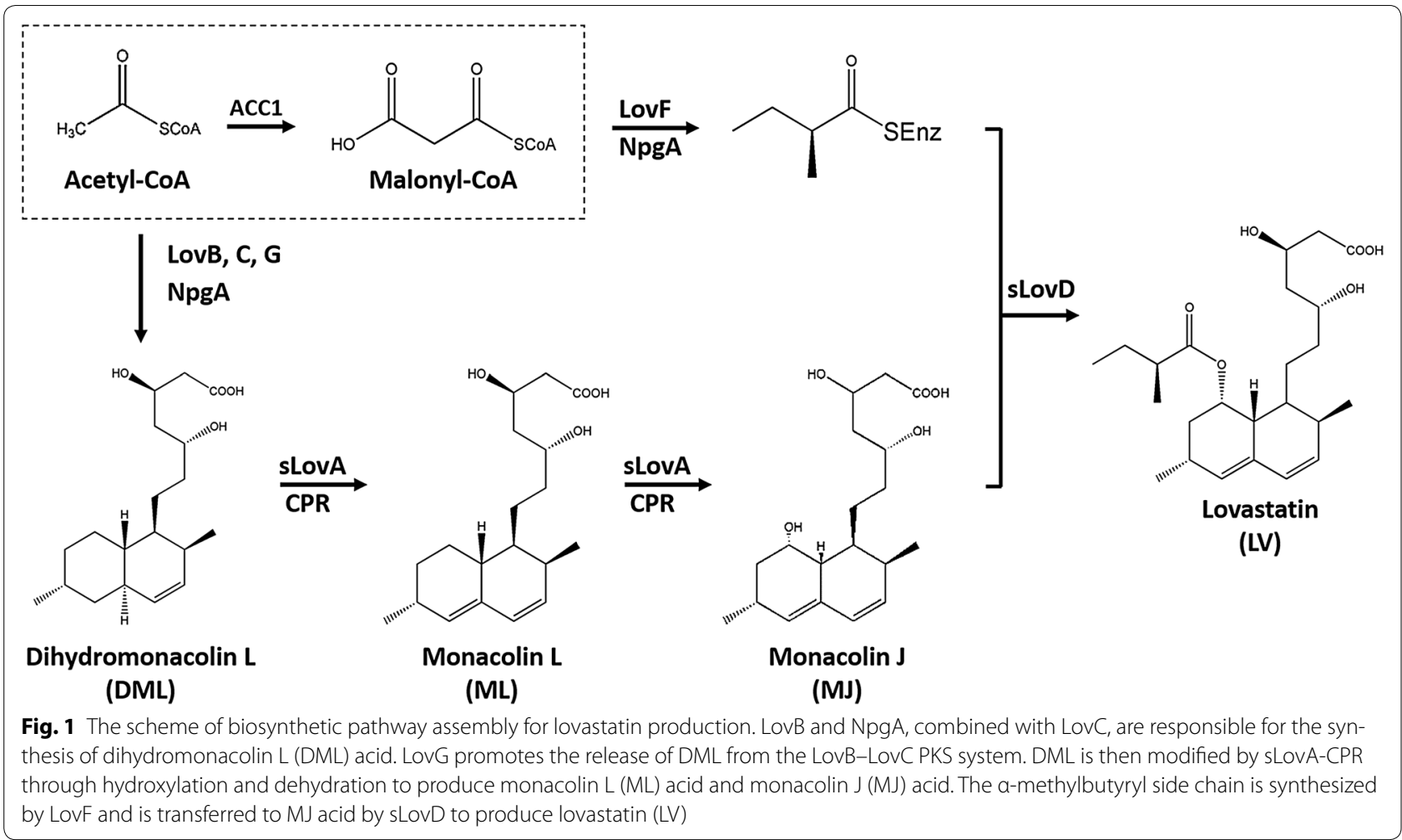

vector pGAP Z $\alpha$ for $P$. pastoris was linearized by digestion with BspT104I and KpnI to delete the $\alpha$-factor. The tap $A$ gene was amplified by overlap PCR from the $A$. terreus $\mathrm{NIH} 2624$ genome as a template, and the introns were removed. The multiple fragments and linearized vector were assembled by ClonExpress ${ }^{\mathrm{TM}}$ II One Step Cloning Kit (Vazyme Biotech Co., Ltd., China) to generate plasmid pZ_P ${ }_{G A P}$-tapA. The same methods were applied to construct plasmids $\mathrm{pZ}_{-} \mathrm{P}_{G A P^{-}} g f p$, and $\mathrm{pZ}$ $\mathrm{P}_{G A P^{-}}$tapA-gfp. These plasmids were linearized by $B \ln \mathrm{I}$ digestion and transferred into $P$. pastoris GS115 to obtain strains GS-P $\mathrm{P}_{G A P^{-}} g f p$, GS-P $\mathrm{P}_{G A P^{-}}$tapA-gfp, and GS-P $\mathrm{P}_{G A P^{-}}$ tapA, respectively. Commercialized vector pPIC 6A for $P$. pastoris was linearized by digestion with EcoRI and SalI; tapA was amplified and ligated with the linearized pPIC 6A to generate plasmid p6A_P $\mathrm{P}_{A O X 1^{-}}$-tapA, which contains the expression cassette under the control of the $A O X 1$ promoter $\left(\mathrm{P}_{A O X 1}\right)$. The expression vector was linearized by PmeI digestion and introduced into $P . p / \mathrm{LV} \_\mathrm{V} \# 9$ to obtain the $P$. $p /$ LV_V\#9-TapA strain. Plasmids and strains used in this study are listed in Additional file 1: Tables S1 and S2.

\section{Phylogenetic, fluorescence-microscopic, and susceptibility analyses}

The phylogenetic tree and bootstrap values were calculated by the neighbor-joining method implemented in
MEGA 7, according to the alignment of the amino acid sequences in the ClustalX software. The distance between branch points showed relatedness of the sequences. The amino acid sequence was BLASTed in the NCBI database. Proteins used to construct the phylogenetic tree are listed in Additional file 1: Table S3. Fluorescence microscopy of the constructed strains $\left(\mathrm{GS}_{-} \mathrm{P}_{G A P}\right.$-tapA$g f p$ and GS_P $\left.{ }_{G A P}-g f p\right)$ was carried out under the inverted microscope DMI3000B (Leica) with a $100 \times$ oil immersion objective. Images were processed using the Leica application suite (version 2.8.1). For susceptibility assays on solid media, strains were grown in the YPD medium until optical density at $600 \mathrm{~nm}\left(\mathrm{OD}_{600}\right)$ of 6 , washed with sterile water, and then equal volumes of cell suspensions were spotted at four dilutions $\left(\mathrm{OD}_{600}=1,0.1,0.01\right.$, and 0.001 ) onto the YPD medium containing $0,0.2,0.5$, or $1.0 \mathrm{mM}$ lovastatin, respectively. The plates were cultured at $30{ }^{\circ} \mathrm{C}$ for 2 days and examined. For the susceptibility assay in a liquid medium, strains were grown in the YPD medium until $\mathrm{OD}_{600}$ of 6 and washed with sterile water. The washed strains were inoculated in the YPD liquid medium containing $0,0.5$, or $1.0 \mathrm{mM}$ lovastatin separately. The initial cell density was maintained at $\mathrm{OD}_{600}=0.5$, and $2 \mathrm{~mL}$ of $50 \%(\mathrm{w} / \mathrm{v})$ glucose was added every $24 \mathrm{~h}$. Broth samples $(1 \mathrm{~mL})$ were collected at $24-\mathrm{h}$ intervals (before and after the glucose feeding) to measure cell growth. Statistical analysis was performed on 
three replicates, and the mean and standard deviation (SD) were calculated and displayed in the figures. Each experiment in this analysis section was conducted three times.

\section{Measurement of concentrations of glucose, ethanol, and acetate}

A biosensing analytical instrument (SBA-4AE) from the Biology Institute of Shan Dong Academy of Sciences was used to determine the concentrations of glucose and ethanol. Standard solutions of glucose $(1 \mathrm{~g} / \mathrm{L})$ and ethanol $(1 \mathrm{~mL} / \mathrm{L})$ were mixed and set to $100 \%$ in the calibration procedure. Acetic acid was analyzed by enzymatic assays (Megazyme, Ireland). Broth samples $(1 \mathrm{~mL})$ were collected and centrifuged at $4{ }^{\circ} \mathrm{C}$ and $12,000 \mathrm{~g}$ for $2 \mathrm{~min}$ to obtain the supernatant, which was then diluted and assayed. Three replicates were analyzed; the mean with SD of the concentrations were calculated and displayed in figures. Measurement of the concentrations of glucose, ethanol, and acetate was conducted two times.

\section{Fermentation in a shake flask}

For shake flask culture, $20 \mu \mathrm{L}$ of the strain (stored at $-80^{\circ} \mathrm{C}$ ) was inoculated into $2 \mathrm{~mL}$ of the YPD medium in a 20 -mL serum bottle and grown at $30{ }^{\circ} \mathrm{C}$ for 2 days at $200 \mathrm{rpm}$ for strain activation. Then, $200 \mu \mathrm{L}$ of the cell broth was inoculated into $50 \mathrm{~mL}$ of the YPD medium in a $250-\mathrm{mL}$ shake flask and grown overnight at $30{ }^{\circ} \mathrm{C}$ and $200 \mathrm{rpm}$ to prepare the first seed culture. Next, $200 \mu \mathrm{L}$ of the first seed culture was inoculated into $50 \mathrm{~mL}$ of the YPD medium in a $250-\mathrm{mL}$ shake flask and grown overnight to $\mathrm{OD}_{600}$ of 6 to prepare the second seed culture. A calculated amount of the second seed culture was centrifuged at $4{ }^{\circ} \mathrm{C}$ and $5000 \mathrm{~g}$ for $10 \mathrm{~min}$ to harvest the cells, which were washed in the YNB medium with two further cycles of centrifugation $\left(4{ }^{\circ} \mathrm{C}, 5000 \mathrm{~g}\right)$. After that, the cells were resuspended in the YNB medium and inoculated into the YNM medium to final density for cultivation $\left(\mathrm{OD}_{600}=1.0\right)$. Methanol was added to the YNM medium at $0.5 \%(\mathrm{v} / \mathrm{v})$ every $24 \mathrm{~h}$. Broth samples $(2 \mathrm{~mL})$ were collected at 24-h intervals to measure the cell growth and metabolite concentrations. Statistical analysis was performed on three replicates; the mean with SD was calculated. Experiments for testing the production of the DML-, MJ- and LV-producing strains were conducted two times.

\section{Extraction and identification of lovastatin and intermediates}

Pichia pastoris culture broth was extracted with two volumes of ethyl acetate with vortex mixing for $5 \mathrm{~min}$. The organic phase was evaporated under reduced pressure, and the residue was dissolved in methanol. The extracts were analyzed by high-performance liquid chromatography (HPLC) on a C18 column (Kromasil ${ }^{\mathrm{TM}}$, Sweden, $250 \mathrm{~mm} \times 4.6 \mathrm{~mm} \times 5 \mathrm{~mm}, 100 \AA$ spherical silica) with a gradient elution strategy at $1 \mathrm{~mL} / \mathrm{min}$ and detection by UV absorbance at $238 \mathrm{~nm}$ (for monacolin L, monacolin $\mathrm{J}$, and lovastatin) and by an evaporative light scattering detector operated at $85{ }^{\circ} \mathrm{C}$ and $2 \mathrm{~mL} / \mathrm{min}$ (for DML). In both elution procedures, the mobile phase was composed of HPLC-grade $\mathrm{H}_{2} \mathrm{O}$ containing $0.1 \%(\mathrm{v} / \mathrm{v})$ acetic acid (solution A) and HPLC-grade acetonitrile (solution B). The sample was subjected to an elution gradient with a mobile phase comprising $35-85 \%(\mathrm{v} / \mathrm{v})$ acetonitrile for $25 \mathrm{~min}$ followed by $85-100 \%(\mathrm{v} / \mathrm{v})$ acetonitrile for $5 \mathrm{~min}$. The final product, lovastatin, was confirmed by liquid chromatography with mass spectrometry (LC-MS; Agilent 1290 Infinity LC and 6230 TOF LC/MS) and nuclear magnetic resonance (NMR) spectroscopy (BRUKERAM-400-spectro) of a freeze-dried sample dissolved in deuterated dimethyl sulfoxide for ${ }^{1} \mathrm{H}$ NMR analysis. The ${ }^{1} \mathrm{H}$ NMR results were then compared with previously reported data (Barriuso et al. 2011).

\section{Bioreactor fermentation}

This fermentation was conducted in a 5-L stirredtank bioreactor (Shanghai Guoqiang Bioengineering Equipment Co., Ltd.) equipped with online sensors for dissolved oxygen (DO), $\mathrm{pH}$, and temperature. The fermentation medium and control process were adapted from the Pichia Fermentation Process Guidelines (PFPG, Invitrogen). The strains were cultured overnight in YPD until the cell density reached $\mathrm{OD}_{600}$ of 6 to prepare the first seed culture. The latter was inoculated into the MGY medium until it reached $\mathrm{OD}_{600}$ of 6 and then served as the second seed culture; $300 \mathrm{~mL}$ of it was aseptically transferred into the bioreactor.

For bioprocess control, a batch phase involving the fermentation basal salt medium containing $40 \mathrm{~g} / \mathrm{L}$ glycerol (PFPG, Invitrogen; Liu et al. 2018) was first conducted and maintained for approximately $18 \mathrm{~h}$. A glycerol-fed batch phase was next carried out with a batch using $50 \%(\mathrm{w} / \mathrm{v})$ glycerol for approximately $12 \mathrm{~h}$, followed by a period of $0.5 \mathrm{~h}$ without any feeding, to allow the glycerol to be fully consumed. The methanol feed was implemented via a gradual increase in the methanol feeding rate from 2.4 to $24 \mathrm{~g} / \mathrm{h}$ during the first $7.5 \mathrm{~h}$, to allow the cells to adapt to methanol catabolism; after that, a constant rate of $24 \mathrm{~g} / \mathrm{h}$ was maintained until the end of the experiment. DO levels reflect the remaining amount of a carbon source in a bioreactor; byproduct ethanol or acetate can also be utilized as carbon sources by $P$. pastoris. If these carbon sources highly accumulated in the broth, DO would remain at low levels because utilization of a carbon source consumes $\mathrm{O}_{2}$. In contrast, when 
the carbon sources were fully consumed, the DO would skyrocket. Following this strategy, we restrictedly controlled the feeding rate of glycerol and methanol according to DO levels and kept all carbon sources completely consumed. Broth samples $(2 \mathrm{~mL})$ were collected at 8 -h intervals and analyzed twice to measure the cell growth and concentrations of the metabolites. The bioreactor fermentation experiments were conducted two times.

\section{Results and discussion}

\section{Assembly of an enhanced biosynthetic pathway} of lovastatin

Plasmid pZ_BCGN was linearized, transferred into the wild-type strain, and employed to screen the microbes for DML-overproducing strains. As shown in Fig. 2a, b, strain P. $p / \mathrm{DML}$ D\#9 grown at $2.0 \mathrm{mg} / \mathrm{mL}$ Zeocin produced the highest concentration of DML $(73.8 \pm 1.8 \mathrm{mg} / \mathrm{L})$, and the production of DML increased as the Zeocin concentration was increased. Moreover, strain P. p/DML_D\#9 grew well in shake flask culture (Additional file 1: Figure S1a) and thus acted as a recipient strain for transformation with a second plasmid (pK_sAR) to generate MJoverproducing strains. The resulting strain $P$. p/MJ_J\#9 grown at $2.0 \mathrm{mg} / \mathrm{mL}$ G418 showed the highest MJ titer $(28.4 \pm 1.3 \mathrm{mg} / \mathrm{L}$; Fig. 2a, c), and its growth was not obviously affected (Additional file 1: Figure S1b). Similarly, the MJ titer also increased as the G418 concentration was increased; however, the obtained improvement was somewhat limited, while the intermediate monacolin L (ML) accumulated remarkably during culture (Fig. 2a). A third plasmid, pG_sDF, was then transferred into $P$. p/MJ_J\#9, and the transformants were screened against different concentrations of hygromycin to find lovastatin-producing strains. Finally, a strain $\left(P . p / L_{-}\right.$V\#9) producing lovastatin at $19.4 \pm 1.7 \mathrm{mg} / \mathrm{L}$ was obtained at $5.0 \mathrm{mg} / \mathrm{mL}$ hygromycin (Fig. 2a, d); this productivity was $592 \%$ higher than that of strain $P . p / L V \_V \# 1$ selected at $0.75 \mathrm{mg} / \mathrm{mL}$ hygromycin. The growth of these lovastatin-producing strains in shake flask culture appeared unaffected by the different concentration of antibiotics (Additional file 1: Figure S1c). Results of LC-MS and NMR analyses confirmed the module weight and structure of the product (lovastatin) produced by $P . p / \mathrm{LV}_{-} \mathrm{V} \# 9$ (Additional file 1 : Figure S2). The $\mathrm{pH}$ values in the culture medium of lovastatin-producing strains were measured and are shown in Additional file 1: Figure S3; these levels similarly dropped from 5.5 to approximately 2.5 , and final $\mathrm{pH}$ did not show a large difference among the lovastatin-producing strains. These results revealed that increasing the gene dosages by strengthening antibiotic stress during pathway assembly efficiently enhanced the production of certain compounds in lovastatin biosynthesis. Nevertheless, excessive expression of heterologous genes usually leads to severe metabolic stress on the host, and the increased intracellular accumulation of target compounds may be harmful to the cell metabolism simultaneously. This problem may be inapparent during shake flask culture, but it will be conspicuous at a bioreactor fermentation level.

\section{Enhancement of cell tolerance to lovastatin by identification and overexpression of a statin pump protein}

Exogenous lovastatin was proved to inhibit yeast cell growth (Ley et al. 2015), whereas excessive intracellular accumulation of lovastatin may also be toxic and impose a metabolic burden on the host cell. Therefore, pumping intracellular lovastatin out of the P. pastoris cell through an efflux protein may relieve the toxicity effect and metabolic stress; as a consequence, the productivity of yeast cells may improve too. For this purpose, we conducted a BLAST search of the NCBI database for statin pump protein MlcE of P. citrinum (Ley et al. 2015); this search retrieved a hypothetical protein from $A$. terreus NIH2624, which was designated as TapA, consisted of 542 amino acid residues, and shared 69\% sequence similarity with MlcE (Additional file 1: Figure S4). Phylogenetic analysis revealed that TapA and MlcE belong to a group of drug resistance proteins of the major facilitator superfamily (MFS): a large and diverse group of secondary transporters (Fig. 3a). The results indicated that TapA may be a statin efflux pump protein of the native lovastatin-producing strain of $A$. terreus and may improve the export efflux of lovastatin and, therefore, enhance the tolerance of $P$. pastoris to lovastatin. To determine the subcellular localization of TapA in engineered P. pastoris, we tagged TapA with green fluorescent protein (GFP) at its carboxylic terminus and expressed it as a single-copy gene. As a control, GFP was expressed alone using the same promoter and expression cassette of a single-copy gene. Fluorescence microscopy evaluation of the resulting strain, GS_P ${ }_{G A P}-t a p A-g f p$, revealed a ringlike distribution of the fluorescent protein at the periphery of the cells and the vacuole (Fig. 3b). By contrast, in the control strain, GS_P ${ }_{G A P^{-}} g f p$, a uniform cytoplasmic distribution of fluorescence was observed (Fig. 3b). The GFP-tagged TapA localized to the cytomembrane, indicating that TapA is a transmembrane protein, just as MlcE in $S$. cerevisiae (Ley et al. 2015). To test whether TapA could increase $P$. pastoris resistance to lovastatin, tapA was expressed under the control of constitutive promoter $\mathrm{P}_{G A P}$ in the wild-type strain. Strain GS_P ${ }_{G A P}$-tapA was obtained and tested by serial dilution spotting on YPD plates supplemented with different concentration of lovastatin. As shown in Fig. 3c, the TapA-expressing strain showed increased resistance to lovastatin as compared with the wild-type strain. 


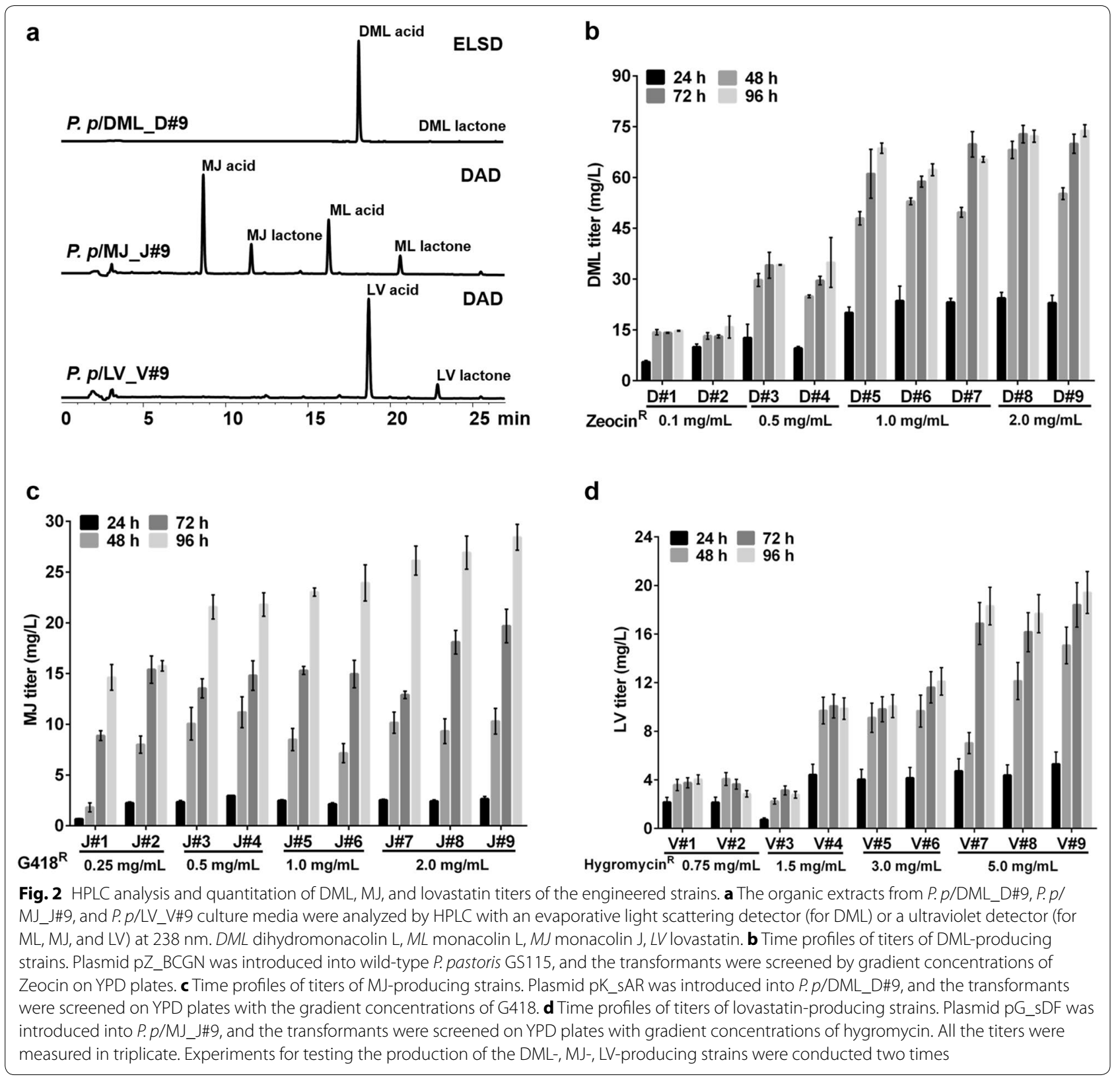

To determine whether this TapA expression strategy could be applied to liquid fermentation, we cultured GS

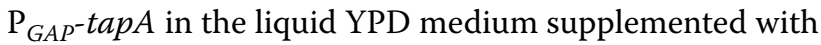
increasing concentrations of lovastatin in shake flask culture. The growth of the wild-type strain was severely inhibited by lovastatin concentrations greater than $0.5 \mathrm{mM}$, whereas the growth of strain $\mathrm{GS}_{-} \mathrm{P}_{\text {GAP }}$-tapA was affected only slightly (Fig. 3d). To avoid interference from the carbon source and byproduct formation, we next tested the concentrations of glucose, ethanol, and acetic acid in the lovastatin susceptibility assay. As shown in Fig. 4a, the glucose concentration in the medium of both strains showed the same trend without extracellular lovastatin feeding. When lovastatin was added at 0.5 or $1.0 \mathrm{mM}$, wild-type strain GS115 consumed less glucose, and residual glucose was more abundant in comparison with strain GS-P ${ }_{G A P}$-tapA. These results confirmed that GS-P ${ }_{G A P}$-tapA expressing the statin pump protein TapA is highly resistant to lovastatin; consequently,

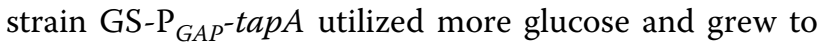
higher cell density (Fig. 3d). Concentrations of ethanol and acetate were measured and are displayed in Fig. 4b, c. In the whole fermentation process, the concentrations of ethanol (Fig. 4b) and acetate (Fig. 4c) did not show a 
difference between strains GS115 and GS-P ${ }_{G A P}$-tapA whether lovastatin was added or not. Ethanol concentration stayed at approximately $0.2 \mathrm{mM}$, while acetic acid concentration stayed at approximately $0.4 \mathrm{mM}$. These results confirmed that formation of byproducts (ethanol or acetate) stayed at low levels and did not cause interference. These data implied that TapA-based resistance in $P$. pastoris may further improve the production of lovastatin in the P. pastoris cell factory.

\section{Improvement in lovastatin production by coexpression of tapA}

To test whether TapA could export the intracellular lovastatin and accordingly improve lovastatin production in yeast, plasmid p6A_P $\mathrm{P}_{A O X 1}$-tapA was linearized and transferred into the multicopy strain $P . p / \mathrm{LV} \_\mathrm{V} \# 9$, thus yielding a tapA-coexpressing strain $P . p / \mathrm{LV} \_\mathrm{V} \# 9$-TapA. Both strains were cultured in the methanol medium (YNM), and a strain of $P . p / \mathrm{LV} \_\mathrm{SC}$ with all genes at single-copy levels (Liu et al. 2018) was cultured as a control. As shown in Fig. 5a, P. p/LV_V\#9-TapA produced $32.9 \pm 1.6 \mathrm{mg} / \mathrm{L}$ lovastatin, which was 69 and $520 \%$ higher than the productivity of $P . p / \mathrm{LV} \_\mathrm{V} \# 9$ and of the single-copy strain (Liu et al. 2018), respectively. The $\mathrm{pH}$ values in the medium of lovastatin-producing strains were nearly the same at the end of fermentation (Additional file 1: Figure S5). Compared with $P . p / \mathrm{LV} \_\mathrm{V} \# 9, P$. $p / \mathrm{LV} \_\mathrm{V} \# 9$-TapA accumulated less intracellular lovastatin a

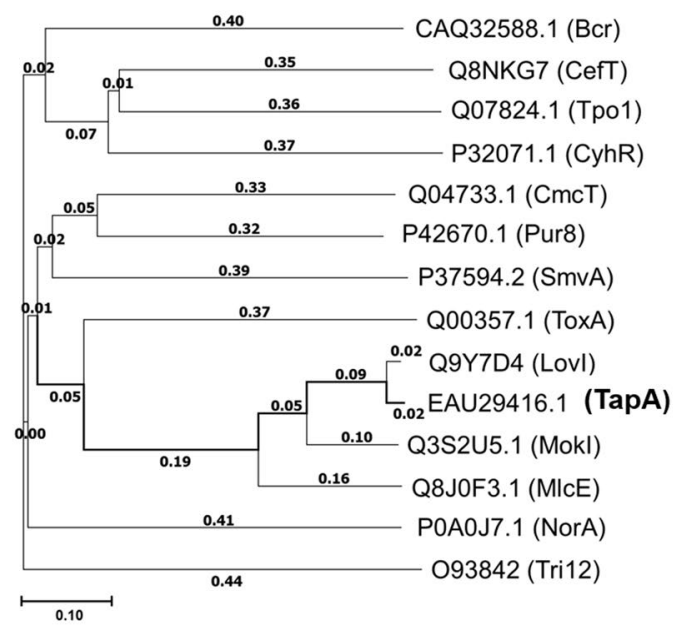

C
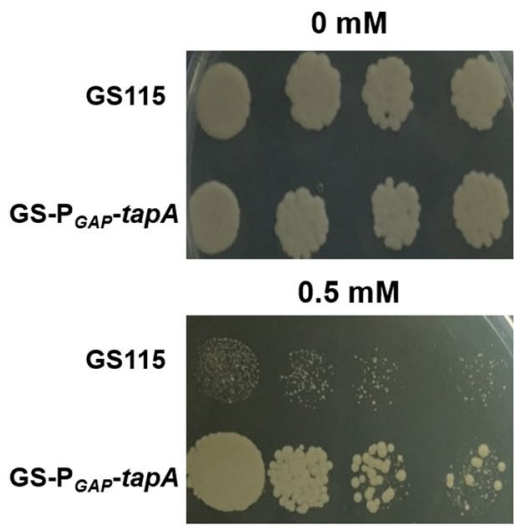

$0.2 \mathrm{mM}$

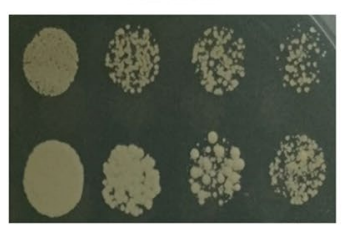

$1.0 \mathrm{mM}$

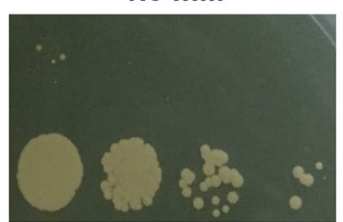

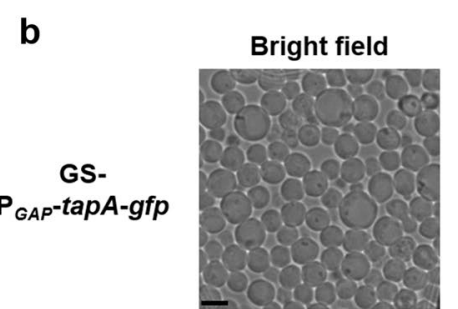
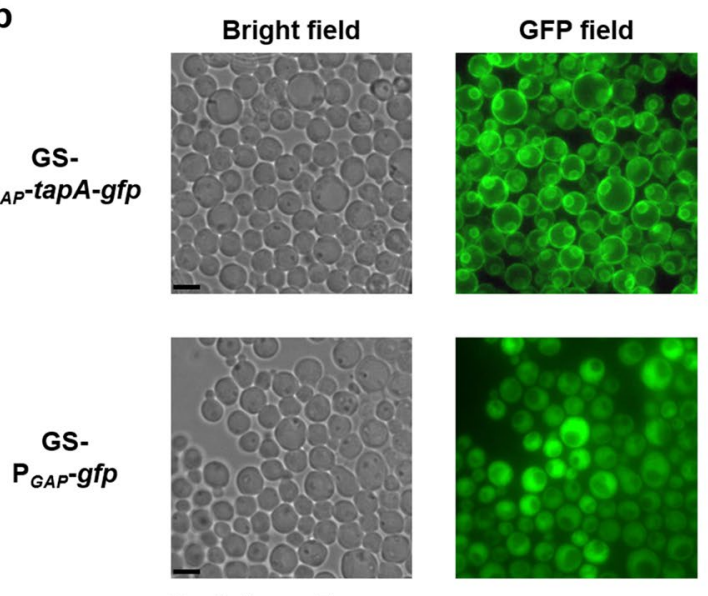

d

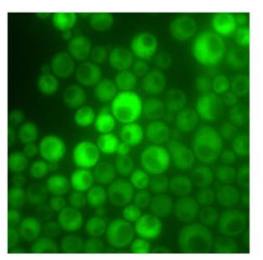

Scale bar $=5 \mu \mathrm{m}$

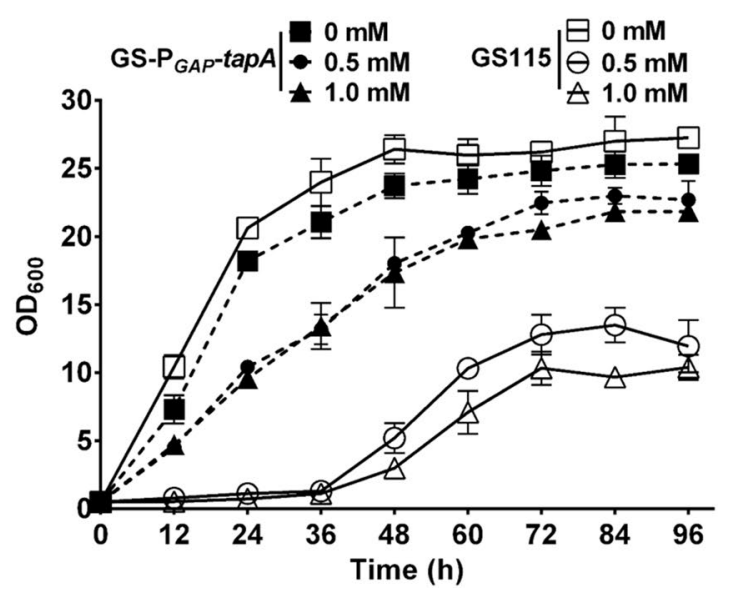

Fig. 3 Phylogenetic and functional analyses of TapA in P. pastoris. a Phylogenetic clustering of putative statin efflux pumps (TapA) with major facilitator superfamily (MFS) transporters involved in the efflux of toxic compounds. Branch TapA is boldfaced, and the GenBank accession numbers and protein names of the sequences are shown. The length of the scale bar is equivalent to 0.1 substitutions of amino acids per site. $\mathbf{b}$ Subcellular localization of TapA in P. pastoris. c Growth comparison of GS_P GAP-tapA and wild-type P. pastoris GS115 on YPD plates containing gradient concen-

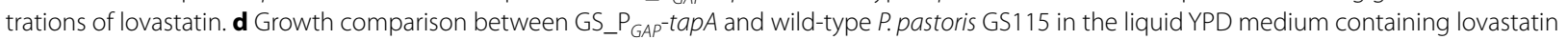
in shaking flasks. Three replicates were tested and experiments were conducted three times 


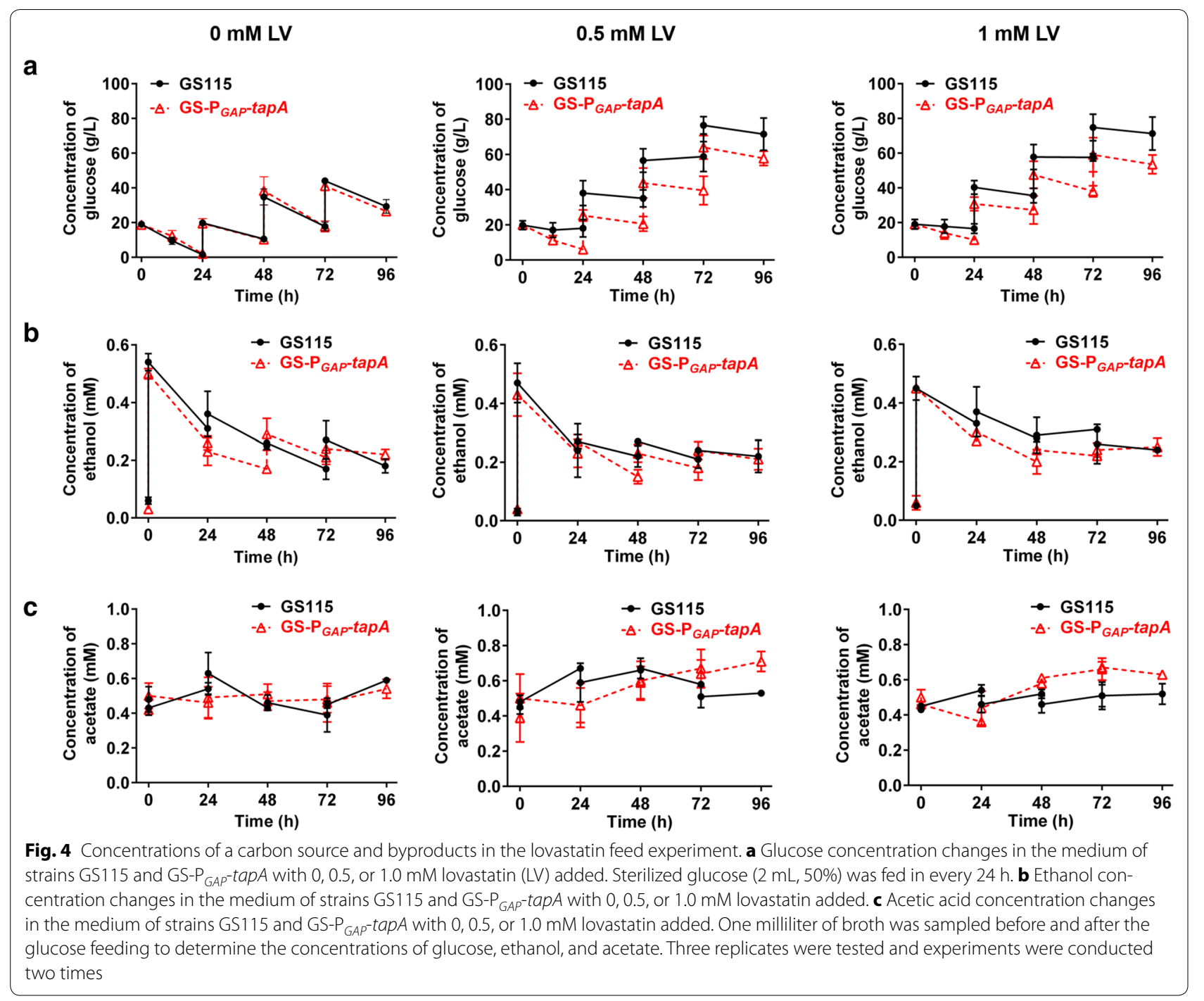

during cultivation (Fig. 5b), suggesting that TapA effectively pumped lovastatin out of the cell.

To further investigate the effects of TapA on lovastatin production, scaled-up experiments on both strains were conducted in a 5 - $\mathrm{L}$ bioreactor. To this end, $3 \mathrm{~L}$ of basal salt medium broth (PFPG, Invitrogen) was used, $\mathrm{pH}$ was controlled at 4.5-5.0, and DO was maintained between 30 and $80 \%$ during the whole fermentation process (Additional file 1: Figure S6). P. $p / \mathrm{LV} \_\mathrm{V} \# 9$ produced $287.5 \pm 2.0 \mathrm{mg} / \mathrm{L}$ lovastatin after $84 \mathrm{~h}$ (Fig. $5 \mathrm{c}$ ), which was $267 \%$ higher than the productivity of the strain with a single copy of the biosynthetic genes ( $P$. $p /$ LV_SC), and even $15 \%$ higher than the productivity of the optimal pathway splitting and coculture strategy applied to the strains with a single copy of biosynthetic genes (Liu et al. 2018). Coexpression of TapA highly improved the production of lovastatin, to $419.0 \pm 9.5 \mathrm{mg} / \mathrm{L}$, after $84 \mathrm{~h}$, which was $46 \%$ higher than the production by $P . p / L V \_V \# 9$. In addition, the yield per cell showed a similar trend, with a $39 \%$ increase. Because both strains expressed numerous heterologous genes, the cell wet weight decreased as the pathway startup was induced by methanol owing to metabolic stress from protein expression and biosynthesis of heterologous compounds. Nevertheless, strain $P . p /$ LV_V\#9-TaP carrying the statin pump protein grew better than P. $p / \mathrm{LV} \_$V\#9 did (Fig. $5 \mathrm{c}$ ), indicating that lovastatin efflux by TapA partially relieved the inhibition of cell growth and effectively improved lovastatin production.

Although P. pastoris has been recognized and widely used as a heterologous protein expression system (Ahmad et al. 2014; Yang and Zhang 2017), application of this classic microbe to reproduce medicinal chemicals via complicated biosynthetic pathways from methanol has seldom been reported. This work shows a strategy of de novo production of lovastatin from methanol in the methylotrophic yeast $P$. pastoris as a host. Considering 
a

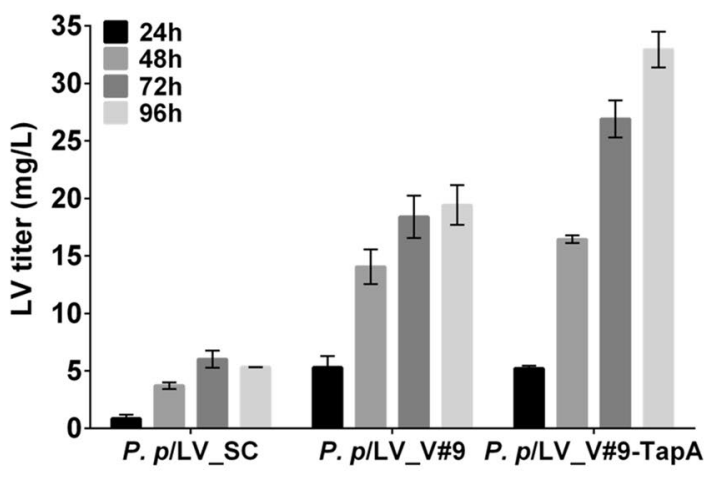

b

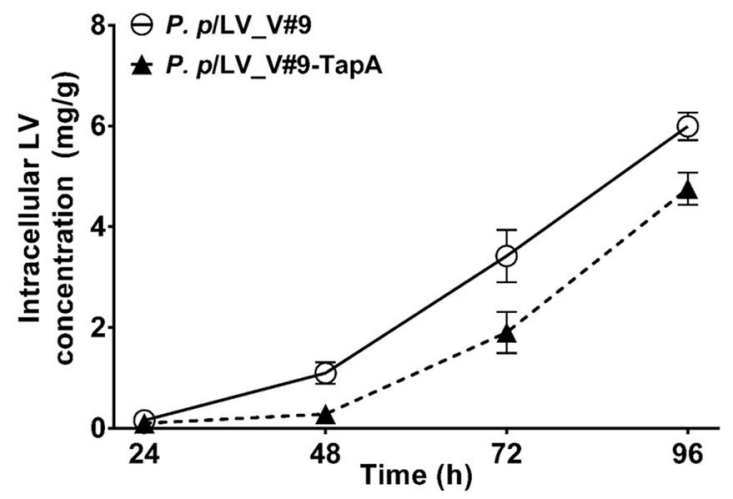

C
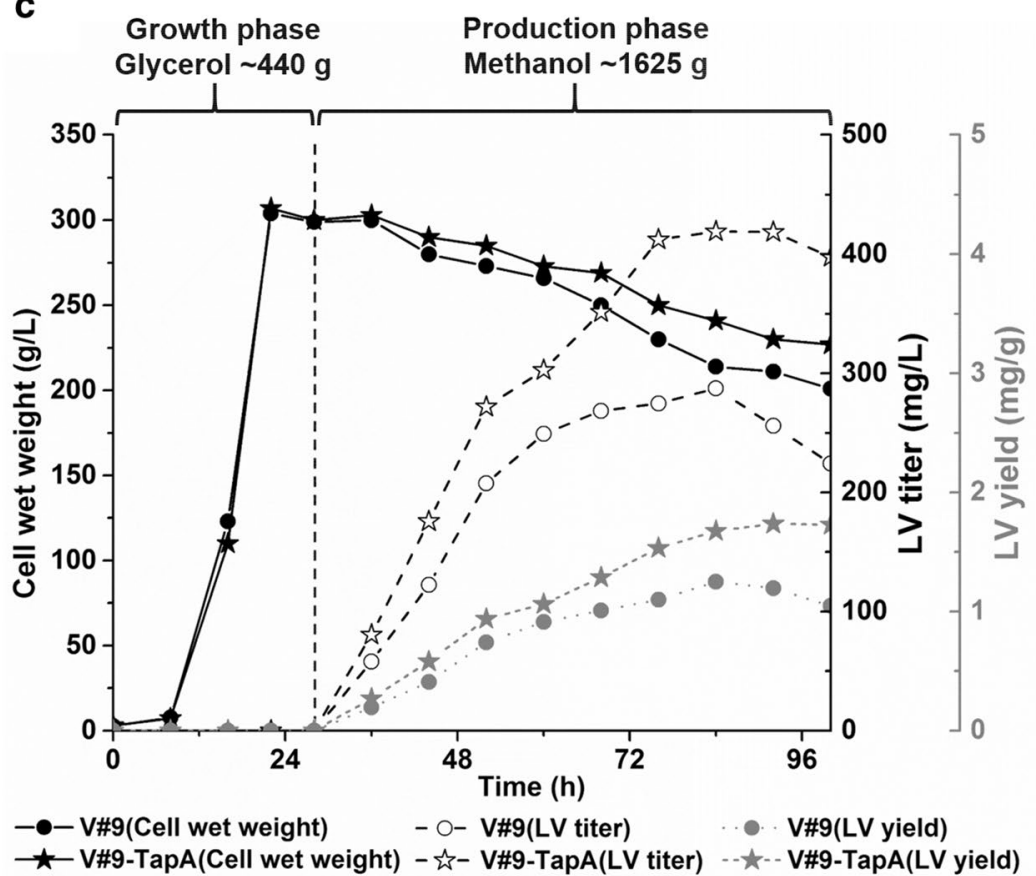

Fig. 5 Coexpression of tapA improved lovastatin production in a shake flask and bioreactor. a Comparison of lovastatin titers between strains P. p/ LV_V\#9 and P.p/LV_V\#9-TapA. b Intracellular lovastatin concentration changes in P.p/LV_V\#9 and P.p/LV_V\#9-TapA. c P.p/LV_V\#9 and P.p/LV_V\#9TapA were employed for fermentation in $3 \mathrm{~L}$ of basal salt medium broth in a 5 - $\mathrm{L}$ bioreactor. Broth samples (2 mL) were collected at 8-h intervals and were analyzed twice to quantify cell growth and lovastatin concentration. Culture methods were the same for both strains. The fermentation experiments in bioreactors were conducted two times

its particular advantages in methanol utilization and protein expression, P. pastoris is expected to be developed into a promising microorganism for the synthesis of bulk pharmaceuticals from a potentially renewable and low-cost feedstock, methanol. Meanwhile, a lovastatin-transporting membrane protein was identified in the industrial host $A$. terreus. Although a similar efflux protein has been recently reported to improve yeast resistance to external lovastatin (Ley et al. 2015), a concrete function of the pump protein was not completely revealed. Here, the ability of this statin pump protein to export intracellular lovastatin was confirmed via coexpression in a lovastatin-producing strain, and its capacity for improving lovastatin production was verified as well. Nevertheless, lovastatin production in this study is still far below the titer of the industrial lovastatin-producing A. terreus strain, which generates $14.26 \mathrm{mM}(\sim 6.1 \mathrm{~g} / \mathrm{L})$ lovastatin after 8 days of fermentation (Huang et al. 2017). The highest heterologous production of lovastatin was $250.8 \mathrm{mg} / \mathrm{L}$ in a $P$. pastoris $-P$. pastoris coculture system in our previous work (Liu et al. 2018). Although we improved lovastatin production to $419.0 \pm 9.5 \mathrm{mg} / \mathrm{L}$ after 
$84 \mathrm{~h}$ here, further research is necessary to determine whether this approach is suitable for industrial production of lovastatin, and this work is still an academic study. Consequently, extensive research into systematic metabolic engineering and bioprocess development needs to be conducted. For instance, $\operatorname{tap} A$ was originally cloned from $A$. terreus, and codons could be optimized for higher expression of TapA in P. pastoris. Pathway splitting and coculture could be combined with an increase in gene dosage and modification and expression of a statin pump protein in the future, and this strategy may further improve lovastatin production. The bioreactor fermentation process here is derived from the manual for basal protein expression in P. pastoris (Invitrogen). Bioprocess development, such as control of the methanol feeding rate or cell growth, will certainly improve lovastatin production.

\section{Conclusions}

A gene dosage increase driven by antibiotic stress in pathway assembly efficiently enhanced lovastatin production from methanol in P. pastoris. A statin pump protein was identified in a native lovastatin-producing microbe, and membrane localization of the new pump in engineered P. pastoris was confirmed. Coexpression of the pump protein enhanced cell resistance to lovastatin, strengthened the intracellular lovastatin efflux, and improved the production of lovastatin in $P$. pastoris. These results also proved that $P$. pastoris is a promising chassis host for heterologous biosynthesis of natural products from the onecarbon substrate, methanol.

\section{Additional file}

Additional file 1. Additional figures and tables.

\section{Abbreviations \\ P. pastoris: Pichia pastoris; DML: dihydromonacolin L; ML: monacolin L; MJ: monacolin J; LV: lovastatin.}

\begin{abstract}
Authors' contributions
$M C$ conceived the project and supervised the research. $Y L, C B$, and $M C$ were responsible for project planning and experimental design. $Y L$ and $C B$ conducted all the experiments. QX and CB participated in the construction of the strains. $Y L$ and JY participated in the bioreactor fermentation experiments. $Y L, C B$, and $M C$ analyzed the data. $Y L$ wrote the manuscript, $M C$ revised and enhanced the manuscript. All the coauthors contributed to the scientific discussion. All authors read and approved the final manuscript.

\section{Author details}

1 State Key Laboratory of Bioreactor Engineering, East China University of Science and Technology, 130 Meilong Road, Shanghai 200237, China. ${ }^{2}$ Shanghai Collaborative Innovation Center for Biomanufacturing, Shanghai 200237, China.
\end{abstract}

\section{Acknowledgements}

Not applicable.
Competing interests

The authors declare that they have no competing interests.

Availability of data and materials

All data generated during this study are included in this article.

\section{Consent for publication}

The authors approved the consent for publishing the manuscript.

\section{Ethics approval and consent to participate}

We declare that this paper does not report any data collected from humans or animals.

\section{Funding}

This work was supported by Fundamental Research Funds for the Shanghai Science and Technology Innovation Action Plan (Grant number 17JC1402400) and the Talent Program of the School of Biotechnology at East China University of Science and Technology.

\section{Publisher's Note}

Springer Nature remains neutral with regard to jurisdictional claims in published maps and institutional affiliations.

Received: 3 February 2018 Accepted: 28 March 2018

Published online: 17 May 2018

\section{References}

Ahmad M, Hirz M, Pichler H, Schwab H (2014) Protein expression in Pichia pastoris: recent achievements and perspectives for heterologous protein production. Appl Microbiol Biotechnol 98:5301-5317

Barriuso J, Nguyen DT, Li JW, Roberts JN, MacNevin G, Chaytor JL, Marcus SL, Vederas JC, Ro DK (2011) Double oxidation of the cyclic nonaketide dihydromonacolin L to monacolin J by a single cytochrome p450 monooxygenase, lovA. J Am Chem Soc 133:8078-8081

Bennett RK, Steinberg LM, Chen W, Papoutsakis ET (2018) Engineering the bioconversion of methane and methanol to fuels and chemicals in native and synthetic methylotrophs. Curr Opin Biotechnol 50:81-93

Billingsley JM, DeNicola AB, Tang Y (2016) Technology development for natural product biosynthesis in Saccharomyces cerevisiae. Curr Opin Biotechnol 42:74-83

Campbell CD, Vederas JC (2010) Biosynthesis of lovastatin and related metabolites formed by fungal iterative pks enzymes. Biopolymers 93:755-763

Cregg JM, Cereghino JL, Shi J, Higgins DR (2000) Recombinant protein expression in Pichia pastoris. Mol Biotechnol 16:23-52

Galanie S, Thodey K, Trenchard IJ, Interrante MF, Smolke CD (2015) Complete biosynthesis of opioids in yeast. Science 349:1095-1100

Huang X, Liang Y, Yang Y, Lu X (2017) Single-step production of the simvastatin precursor monacolin $\mathrm{J}$ by engineering of an industrial strain of Aspergillus terreus. Metab Eng 42:109-114

Kennedy J, Auclair K, Kendrew SG, Park C, Vederas JC, Hutchinson CR (1999) Modulation of polyketide synthase activity by accessory proteins during lovastatin biosynthesis. Science 284:1368-1372

Ley A, Coumou HC, Frandsen RJN (2015) Heterologous expression of MIcE in Saccharomyces cerevisiae provides resistance to natural and semisynthetic statins. Metab Eng Commun 2:117-123

Liu Y, Tu X, Xu Q, Bai C, Kong C, Liu Q, Peng Q, Zhou X, Zhang Y, Cai M (2018) Engineered monoculture and co-culture of methylotrophic yeast for de novo production of monacolin J and lovastatin from methanol. Metab Eng 45:189-199

Martín JF, Casqueiro J, Liras P (2005) Secretion systems for secondary metabolites: how cells send out messages of intercellular communication. Curr Opin Microbiol 8:282-293

Price JV, Chen L, Whitaker WB, Papoutsakis E, Chen W (2016) Scaffoldless engineered enzyme assembly for enhanced methanol utilization. Proc Natl Acad Sci USA 113:12691-12696

Räuchle K, Plass L, Wernicke HJ, Bertau M (2016) Methanol for renewable energy storage and utilization. Energy Technol 4:193-200 
Ro DK, Paradise EM, Ouellet M, Fisher KJ et al (2006) Production of the antimalarial drug precursor artemisinic acid in engineered yeast. Nature 440:940-943

Schwarzhans JP, Luttermann T, Geier M, Kalinowski J, Friehs K (2017) Towards systems metabolic engineering in Pichia pastoris. Biotechnol Adv 35(6):681-710

Singh SK, Pandey A (2013) Emerging approaches in fermentative production of statins. Appl Biochem Biotechnol 171(4):927-938

Vogl T, Hartner FS, Glieder A (2013) New opportunities by synthetic biology for biopharmaceutical production in Pichia pastoris. Curr Opin Biotechnol 24:1094-1101

Wang F, Lv X, Xie W, Zhou P, Zhu Y, Yao Z, Yang C, Yang X, Ye L, Yu H (2017) Combining Gal4p-mediated expression enhancement and directed evolution of isoprene synthase to improve production in Saccharomyces cerevisiae. Metab Eng 39:257-266
Whitaker WB, Sandoval NR, Bennett RK, Fast AG, Papoutsakis ET (2015) Synthetic methylotrophy: engineering the production of biofuels and chemicals based on the biology of aerobic methanol utilization. Curr Opin Biotechnol 33:165-175

Xie X, Watanabe K, Wojcicki WA, Wang CC, Tang Y (2006) Biosynthesis of lovastatin analogs with a broadly specific acyltransferase. Chem Biol 13:1161-1169

Xu W, Chooi YH, Choi JW, Li S, Vederas JC, Da Silva NA, Tang Y (2013) LovG: the thioesterase required for dihydromonacolin $L$ release and lovastatin nonaketide synthase turnover in lovastatin biosynthesis. Angew Chem Int Ed 52:6472-6475

Yang Z, Zhang Z (2017) Engineering strategies for enhanced production of protein and bio-products in Pichia pastoris: a review. Biotechnol Adv. https://doi.org/10.1016/j.biotechadv.2017.11.002 (in press)

\section{Submit your manuscript to a SpringerOpen ${ }^{\circ}$ journal and benefit from:}

- Convenient online submission

- Rigorous peer review

- Open access: articles freely available online

- High visibility within the field

- Retaining the copyright to your article

Submit your next manuscript at $\boldsymbol{\nabla}$ springeropen.com 